\title{
Remote Sensing Using Canopy and Leaf Reflectance for Estimating Nitrogen Status in Red-blush Pears
}

\author{
Eileen M. Perry ${ }^{1}$ \\ Department of Economic Development, Jobs, Transport and Resources, Cnr \\ Midland Highway \& Taylors Street, Epsom, Victoria 3551, Australia; and \\ Department of Infrastructure Engineering, The University of Melbourne, \\ Parkville, Victoria 3010, Australia
}

Ian Goodwin and David Cornwall
Department of Economic Development, Jobs, Transport and Resources, 255
Ferguson Road, Tatura, Victoria 3616, Australia

Additional index words. spectral reflectance, chlorophyll indices, Pyrus communis

\begin{abstract}
Reflectance measurements at leaf and canopy scales were made in a red-blush pear (Pyrus communis) orchard for two growing seasons. Canopy reflectance measurements were obtained using a multispectral camera flown on board an unmanned aerial vehicle (UAV), and leaf reflectance measurements were undertaken in a laboratory using a portable spectrometer. These measurements were used to compute reflectance indices as surrogates for direct leaf nitrogen $(N)$ concentration measurements. The indices were evaluated against laboratory analysis of leaf $\mathbf{N}$ concentration. Regression results for leaf $\% \mathrm{~N}$ on canopy-level measurements with the multispectral camera resulted in the highest $\boldsymbol{R}^{2}$ value $\left[R^{2}=0.67\right.$; root mean square error $\left.(\mathrm{RMSE})=0.24 \% \mathrm{~N}\right]$ with a new index, Modified Canopy Chlorophyll Content Index (M3CI)_710 $\mathrm{nm}$. Regression results for leaf \% $\mathrm{N}$ on leaf-level measurements in-laboratory resulted in the highest $R^{2}$ value $\left(R^{2}=0.65\right)$ with two other indices, Normalized Difference Vegetation Index (NDVI) and Normalized Difference Red-edge Index (NDRE)_720 nm. The corresponding RMSE values were $0.26 \% \mathrm{~N}$. The results indicate that reflectance indices measured at the leaf level, with a controlled light source and calibration, could be used to estimate leaf \%N. An analysis of uncertainty indicated that if leaf $\% \mathrm{~N}$ is estimated from leaf-level reflectance values, 10 or more leaves (from the same tree) should be averaged. The results support the use of a UAV-based assessment for canopy \% $\mathrm{N}$ using the $\mathrm{M3CI} 710 \mathrm{~nm}$, which could provide spatial information of leaf $\mathrm{N}$ concentration across an orchard.
\end{abstract}

The Australian pear industry produced $105,243 \mathrm{t}$ of fruit in 2015 , at a value of $\$ 125$ million (ABS, 2016a, 2016b); however, this production represents a steady decline since a peak in 2004. Pear breeding programs throughout the world have released new fresh market cultivars that are of premium quality and aim to increase world consumption of pears. Most of the selections are either redblushed (e.g., 'Celina', 'Gem', and 'ANP0131') or full-red skinned cultivars (e.g., PremP009) that are more popular with consumers (Human, 2013). Australian growers, predominantly in the Goulburn Valley of north-central Victoria, are switching to these new cultivars. Moving away from high yields of traditional green-skinned cultivars to these new red-blush cultivars brings new challenges, in particular, the need to manage

Received for publication 8 Aug. 2017. Accepted for publication 13 Nov. 2017.

${ }^{1}$ Corresponding author. E-mail: eileen.perry@ecodev. vic.gov.au.

This is an open access article distributed under the CC BY-NC-ND license (http://creativecommons. org/licenses/by-nc-nd/4.0/). able to accurately, easily, and cheaply measure leaf $\mathrm{N}$ across an entire orchard would be ideal. Whereas nondestructive methods to estimate $\% \mathrm{~N}$ for pear leaves have been presented (Jie et al., 2014), methods to estimate $\% \mathrm{~N}$ at canopy scales for pear orchards have yet to be developed. Previous research (Fitzgerald et al., 2010; Perry et al., 2012) shows that using remote sensing to estimate $\mathrm{N}$ in wheat canopies is effective, and calculation of the Canopy Chlorophyll Content Index (CCCI) from canopy spectral reflectance of wheat showed good correlation with $\mathrm{N}$ uptake ( $\mathrm{kg} \mathrm{N} / \mathrm{ha})$. In this research, we extend the previous research on wheat to redblush pears, acquiring and analyzing datasets of remote sensing measurements and corresponding leaf $\% \mathrm{~N}$ from laboratory analysis to determine the feasibility of remote sensing to characterize $\mathrm{N}$ status. This research also differs from previous research in the use of high spatial resolution imagery from UAV platforms to measure canopy reflectance to estimate leaf $\mathrm{N}$ concentration.

\section{Materials and Methods}

Site. A field trial was established near Tatura, Australia $\left(36.44^{\circ} \mathrm{S} 145.27^{\circ} \mathrm{E} ; 115 \mathrm{~m}\right.$ mean sea level). The site is characterized as having Red Sodosol soils (Isbell, 2002). The region has a temperate climate with warm to hot summers with rainfall tending to be winter dominant. The long-term mean maximum and minimum temperatures in January are 29.3 and $13.9^{\circ} \mathrm{C}$, respectively (Bureau of Meteorology, 2017; station number 81049). The long-term mean summer rainfall is $98 \mathrm{~mm}$ and the mean annual rainfall is $491 \mathrm{~mm}$ (Bureau of Meteorology, 2017). Annual average reference crop evapotranspiration (Allen et al., 1998) is $\approx 1190 \mathrm{~mm}$ (22-year mean, http://www.longpaddock.qld. gov.au/silo/). A red-blush pear (Pyrus communis) orchard was established in Winter 2009 with three 72-m rows of BP1 rootstock planted in Winter 2009 and budded with 'ANP-0131' scion (marketed as Deliza ${ }^{\circledR}$ ) in Spring 2009 at $0.75-$ and $4.0-\mathrm{m}$ tree and row spacing, respectively, and grown on Open Tatura trellis in north-south oriented rows. Trees were microirrigated using turbulent flow jet stream sprayers (Tornado Ray Jet; Rivulis Irrigation Ltd., Gvat, Israel) located midway between every second tree and $\approx 0.3 \mathrm{~m}$ above the soil surface. The micro sprayers delivered $55 \mathrm{~L} \cdot \mathrm{h}^{-1}$ with a surfacewetting pattern of $\approx 4.0 \mathrm{~m}$ diameter. Irrigation was applied for $3-1 / 2 \mathrm{~h}$ about every $3 \mathrm{~d}$ in midsummer to match crop water requirement (Goodwin et al., 2015). Pest and disease protection methods were similar to commercial orchards with regular monitoring to determine spray requirements. The interrows were regularly slashed to maintain a grass sward and the tree rows were sprayed with herbicide as per commercial practice.

Experimental design. Experimental plots were established in 2014, configured as a randomized complete block design with six blocks, and three treatments each, for 
a total of 18 plots. Each plot consisted of three trees, with a minimum of two trees $(1.5 \mathrm{~m})$ as a buffer between plots. The treatments were three levels of fertilizer, applied at nil (control), medium, and high levels to establish a range of leaf $\mathrm{N}$ values. Calcium nitrate $(15 \% \mathrm{~N})$ was applied as a solution in water across the three trees in each plot. Fertilizer applications were made three times during the 2014-15 (year 1, Y1) growing season, 10 times during the 2015-16 (year 2, Y2) growing season, and 11 times during the 2016-17 (year 3, Y3) growing season. The annual totals for the high-treatment plots were 209,280 , and $280 \mathrm{~kg} \mathrm{~N} /$ ha for Y1, Y2, and $\mathrm{Y} 3$, respectively; medium treatments were half the high-treatment levels. Full bloom (for all plots) occurred 23 Sept., 21 Sept., and 27 Sept. for Y1, Y2, and Y3, respectively.

Validation of leaf $\% N$. Leaf $\% \mathrm{~N}$ was determined throughout the experimental period, at dates corresponding to the remote sensing. Leaf samples (20 or more) were collected from the three trees for each plot, following recommendation from Reuter and Robinson (1997). Four midshoot leaves (including petioles) from 20 current season's extension shoots (10 each from east- and west-facing sides of a tree) on each tree were collected and pooled. Leaves were oven dried $\left(90^{\circ} \mathrm{C}\right)$ and sent to a commercial laboratory for sample preparation and leaf $\% \mathrm{~N}$ analysis using Dumas combustion on dried, ground samples.

Canopy reflectance measurements. Canopy reflectance measurements were made using a six-band multispectral camera (Tetracam Micro-MCA, Chatsworth, CA) flown on a multirotor UAV. The camera was configured with spectral bands at 550, 660, $710,720,730$, and $810 \mathrm{~nm}$, all $10-\mathrm{nm}$ wide. Imagery was acquired over two growing seasons: 48, 98, 134, and $218 \mathrm{~d}$ after full bloom (DAFB) in Y2, and 38, 120, and 190 DAFB in Y3. On each of these days, the UAV was flown at $\approx 30 \mathrm{~m}$ above ground level,
A

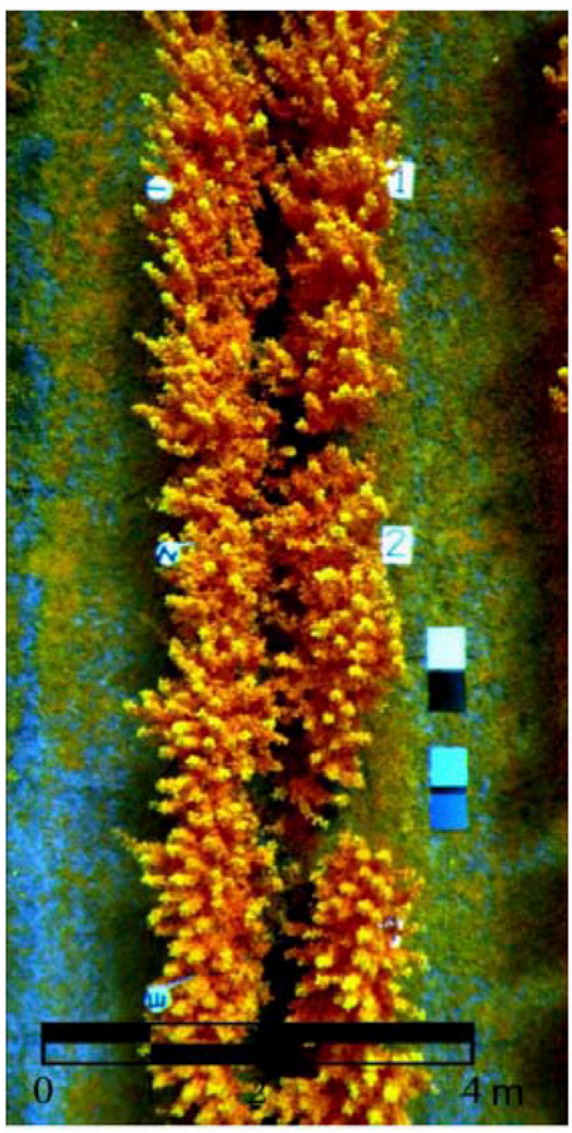

B

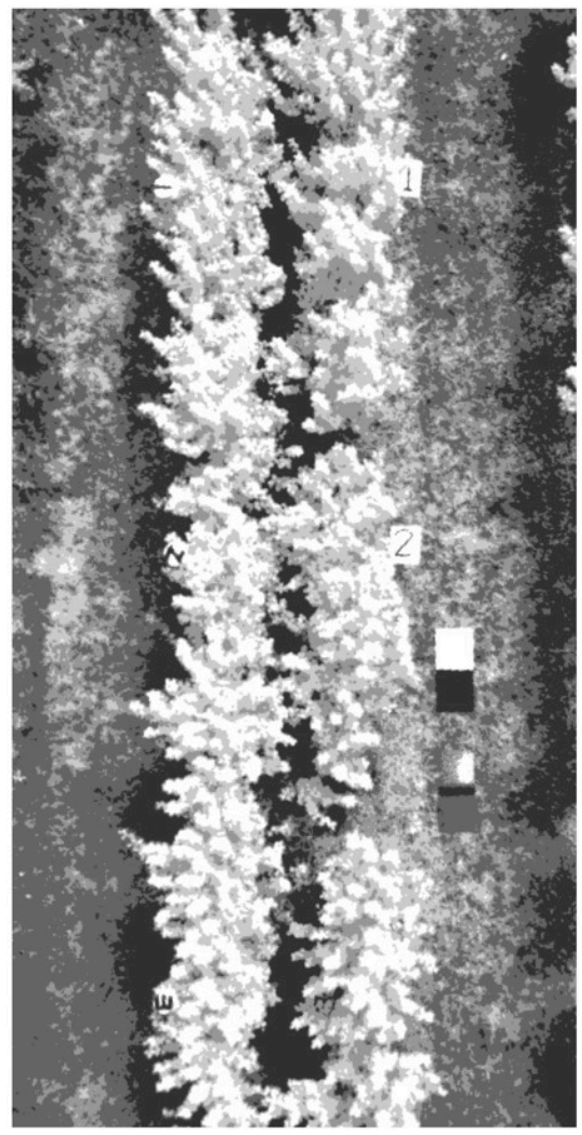

C

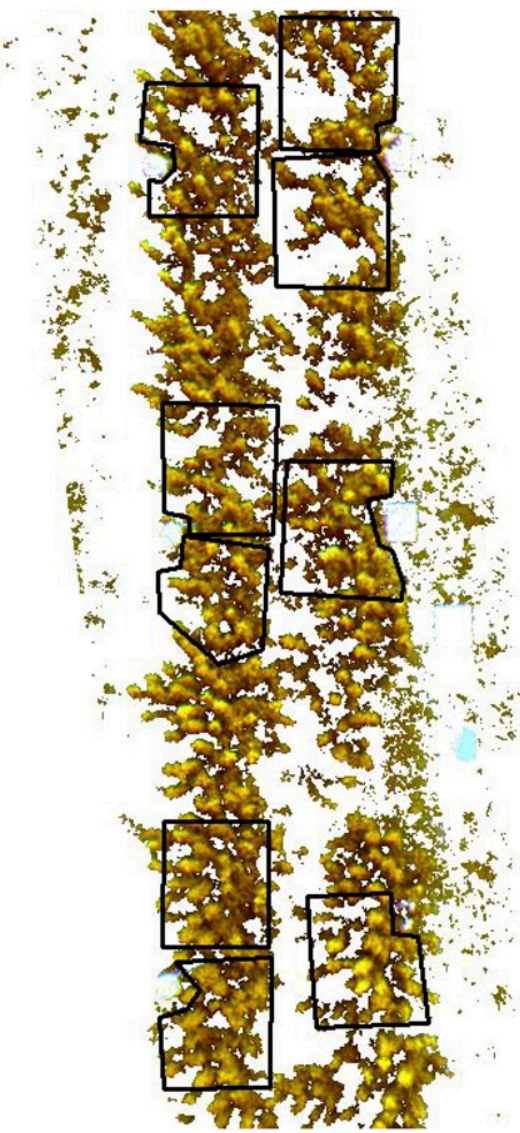

Fig. 1. Example multispectral imagery showing (A) reflectance in false color (810, 710, and $660 \mathrm{~nm}$ displayed as RGB), (B) corresponding ISODATA classification, and (C) masked reflectance and sample areas.

Table 1. Reflectance indices used.

\begin{tabular}{|c|c|c|}
\hline$\overline{\text { Index }}$ & Formula & References \\
\hline Modified Cellulose Absorption Index, MCAI & $0.5\left(R_{2.0 \mu \mathrm{m}}+R_{2.2 \mu \mathrm{m}}\right)-R_{2.1 \mu \mathrm{m}}$ & Daughtry (2001) \\
\hline Chlorophyll Index Red-edge, CI & $\left(R_{\mathrm{NIR}} / N_{\mathrm{RE}}\right)-1$ & Gitelson et al. (2006) \\
\hline $\begin{array}{l}\text { Modified Chlorophyll Absorption Reflectance } \\
\text { Index, MCARI }\end{array}$ & {$\left[\left(R_{700 \mathrm{~nm}}-R_{670 \mathrm{~nm}}\right)-0.2 *\left(R_{700 \mathrm{~nm}}-R_{550 \mathrm{~nm}}\right)\right] *\left(R_{700 \mathrm{~nm}} / R_{670 \mathrm{~nm}}\right)$} & Daughtry et al. (2000) \\
\hline Modified Canopy Chlorophyll Content Index, M3CI & $\left(R_{\mathrm{NIR}}+R_{\mathrm{Red}}-R_{\mathrm{RE}}\right) /\left(R_{\mathrm{NIR}}-R_{\mathrm{Red}}+R_{\mathrm{RE}}\right)$ & This paper \\
\hline Simplified CCCI, SCCCI & NDRE/NDVI & Barnes et al. (2000) \\
\hline Structure Insensitive Pigment Index, SIPI & $\left(R_{800 \mathrm{~nm}}-R_{445 \mathrm{~nm}}\right) /\left(R_{800 \mathrm{~nm}}-R_{680 \mathrm{~nm}}\right)$ & Penuelas et al. (1995) \\
\hline Triangular Greenness Index, TGI & $=-0.5\left[(670-480)\left(R_{670 \mathrm{~nm}}-R_{550 \mathrm{~nm}}\right)-(670-550)\left(R_{670 \mathrm{~nm}}-R_{480 \mathrm{~nm}}\right)\right]$ & Hunt et al. (2013) \\
\hline
\end{tabular}


resulting in pixels $\approx 1 \mathrm{~cm}$. Image acquisitions were timed within $\pm 2 \mathrm{~h}$ of local solar noon. Each of the six blocks (with three plots each) was imaged separately with the UAV hovering over each block. A set of calibration reflectance panels with four grey levels were placed on the ground within each of the six blocks so that every frame (image) included a complete set of panels to convert the imagery to reflectance. Physical markers were used on the ground and canopy to indicate the location of each plot.

Image processing was performed to convert the camera 10 bit raw values to canopy reflectance and extract the reflectance values representing each plot (Fig. 1). Each image was processed and analyzed separately (not "stitched together"). A linear relationship was determined between the known reflectance and raw image values for the four calibration panels for each band in each image ("empirical line fitting"). These equations were used to convert each image to reflectance values. The reflectance images were classified using the ISODATA unsupervised classifier (Ball and Hall, 1965), and the classes representing sunlit tree canopy were used to select canopy pixels from the reflectance imagery. The camera used for this study has separate optics for each band, which combined with the varying distance to target as a result of the irregular canopy, and causes registration errors between bands. To avoid artifacts, polygons were drawn around each plot area, and summary statistics for each band were computed based on the masked reflectance imagery. Mean values for each band were used to compute the reflectance indices. The image processing was performed using Erdas Imagine (Hexagon Geospatial, Madison, AL) and R (R Core Team, 2017).

Leaf reflectance measurements. Leaf reflectance measurements were made to evaluate the same indices determined from the canopy reflectance values in a highly controlled environment. Reflectance measurements were made on 79 and 183 DAFB in Y3 using a portable spectrometer (ASD Field Spec FR; ASD Inc., Boulder, CO) and a leaf clip foreoptic with an internal light source and calibration. Measurements were made within $2 \mathrm{~h}$ of leaf collection, and after reflectance measurements, the leaves were processed and sent to a commercial laboratory for $\mathrm{N}$ analysis. For each plot, five leaves were randomly selected from the 20 or more collected. Three measurements were made per leaf, resulting in 15 measurements per plot.

Reflectance indices. Vegetation indices were computed from the canopy and leaf reflectance measurements. The indices used, along with the corresponding equations and references, are presented in Table 1. Several indices were evaluated for the canopy reflectance measurements, using the available bands of the multispectral camera: CCCI, Simplified Canopy Chlorophyll Content Index (SCCCI), M3CI, Chlorophyll Index Rededge (CI), Modified Chlorophyll Absorption Reflectance Index (MCARI), and NDVI. The first four were evaluated using both the
710- and 720-nm bands. For leaf measurements with the portable spectrometer, additional indices reported to be sensitive to differences in chlorophyll concentration and/or leaf $\mathrm{N}$ were evaluated: Enhanced Vegetation Index, Plant Senescence Reflectance Index (PSRI), Structure Insensitive Pigment Index, and Triangular Greenness Index.

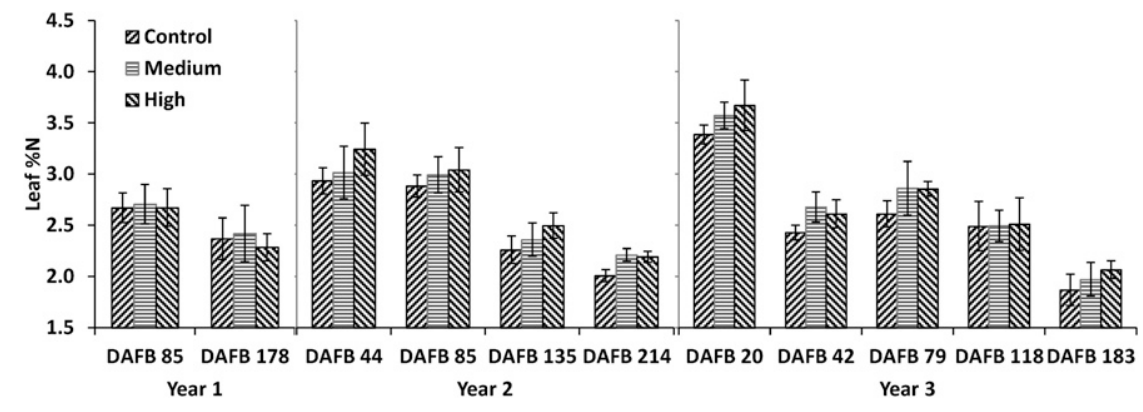

Fig. 2. Mean leaf $\% \mathrm{~N}$ by treatment (Control, Medium, and High) and day after full bloom. Error bars represent \pm SD.

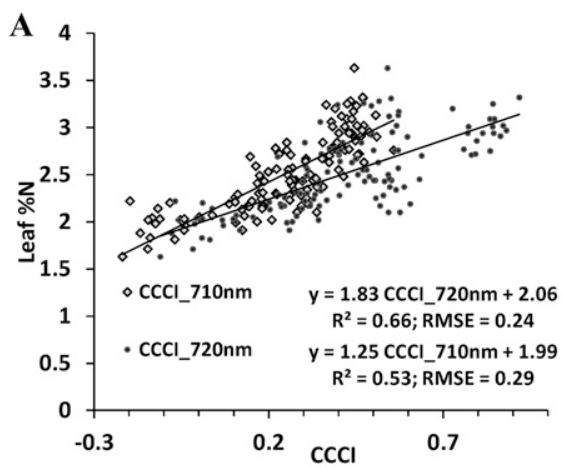

C

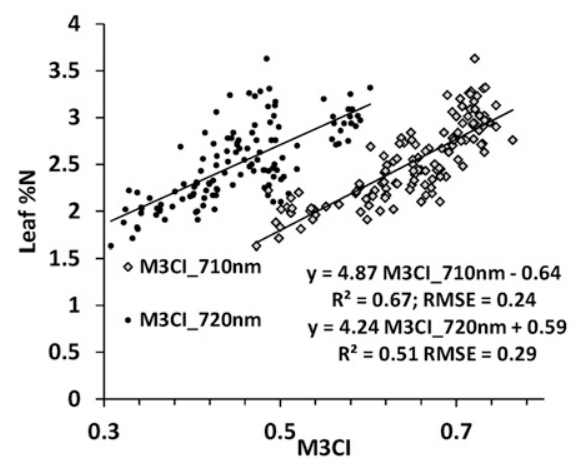

$\mathbf{E}$

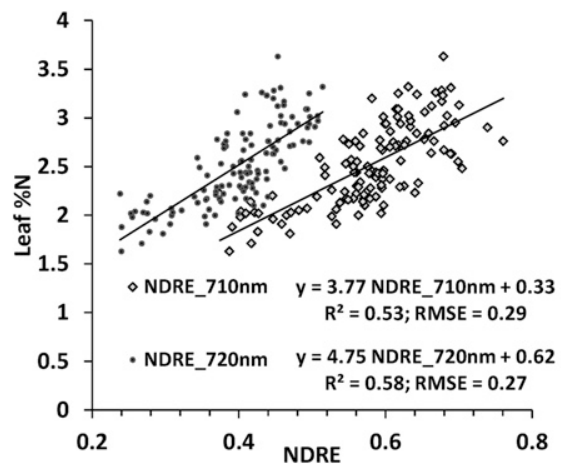

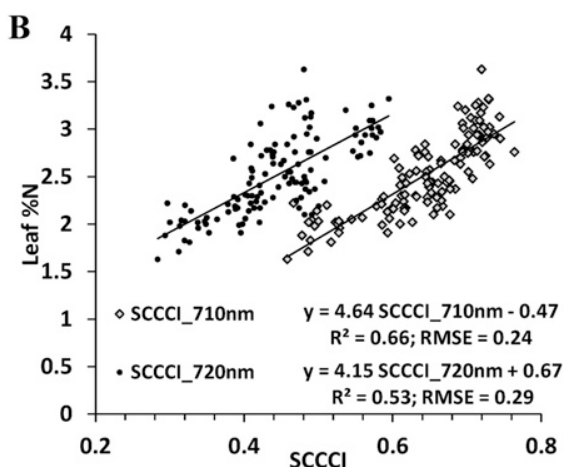

D

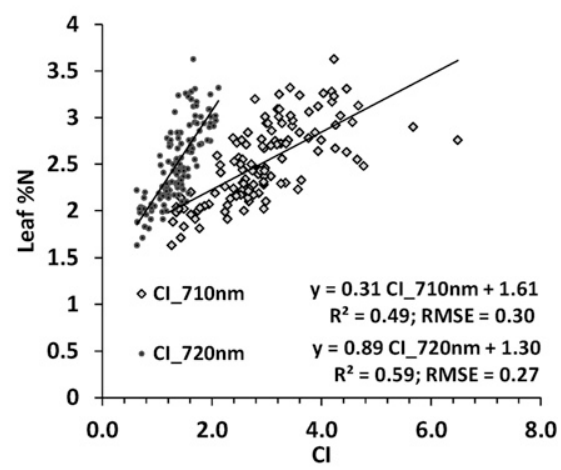

F

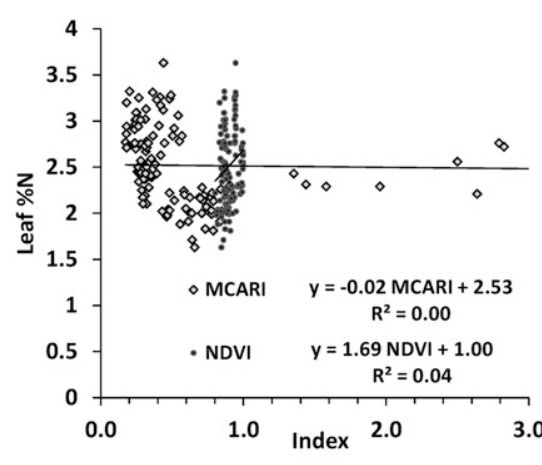

Fig. 3. Measured leaf \%N vs. (A) Canopy chlorophyll content index (CCCI), (B) Simplified Canopy Chlorophyll Content Index (SCCCI), (C) Modified Canopy Chlorophyll Content Index (M3CI), (D) Chlorophyll Index Red-edge (CI), (E) Normalized Difference Red-edge Index (NDRE), and (F) Modified Chlorophyll Absorption Reflectance Index (MCARI) and Normalized Difference Vegetation index (NDVI). The corresponding regression coefficients, adjusted $R^{2}$ values, and root mean square error (RMSE) values are shown in each graph. 
CCCI requires the computation of bounds determined from graphing NDRE vs. NDVI. These bounds were determined for CCCI using the 720-nm band (CCCI_720 nm) from Y2 data. These bounds were similar to those reported by Fitzgerald et al. (2010) for wheat: $\mathrm{NDRE}_{\text {min }}=0.32$ and $\mathrm{NDRE}_{\text {max }}=0.62$ whereas the published values for wheat were 0.34 and 0.61 , respectively. Bounds for CCCI_710 nm were determined on all multispectral camera data. The bounds used were $\mathrm{NDRE}_{\text {min }}=0.54$ and $\mathrm{NDRE}_{\text {max }}=0.94$. SCCCI avoids the use of bounds and is simply the ratio of the computed NDRE over NDVI. To reduce the number of embedded ratios and terms, and therefore the errors propagated through the equation, a further simplification of the CCCI and was developed. The Modified CCCI or M3CI was computed as follows:

$\mathrm{M} 3 \mathrm{CI}=\left(R_{\mathrm{NIR}}+R_{\mathrm{R}}-R_{\mathrm{RE}}\right) /\left(R_{\mathrm{NIR}}-R_{\mathrm{R}}+R_{\mathrm{RE}}\right)$

where $R_{\mathrm{NIR}}$ is measured reflectance in the $810-\mathrm{nm}$ band, $R_{\mathrm{R}}$ is measured reflectance in the $660-\mathrm{nm}$ band, and $R_{\mathrm{RE}}$ is measured reflectance in the 710-nm band (M3CI_710 nm) or 720-nm band (M3CI_720 nm).

Statistical analysis. Relationships between measured leaf $\% \mathrm{~N}$ and the canopy and leaf reflectance indices were determined by linear regression in Genstat 18.1 (VSN International Limited, Oxford, UK).

Error analyses. Error propagation analysis was performed to evaluate the impact of uncertainty in canopy reflectance measurements on estimated leaf $\% / \mathrm{N}$ using $\mathrm{M} 3 \mathrm{CI}$. Leaf $\% \mathrm{~N}$ was computed from M3CI using relationships developed with measured leaf $\% \mathrm{~N}$ (discussed in Results). The errors were propagated through the equation terms using the following rules:

$$
\delta \mathrm{Num}=\operatorname{SQRT}\left(\delta R_{\mathrm{NIR}}{ }^{2}+\delta R_{\mathrm{R}}{ }^{2}+\delta R_{\mathrm{RE}}{ }^{2}\right)
$$

$$
\delta \text { Den }=\operatorname{SQRT}\left(\delta R_{\mathrm{NIR}}{ }^{2}+\delta R_{\mathrm{R}}^{2}+\delta R_{\mathrm{RE}}^{2}\right)
$$

$$
\begin{aligned}
& \delta \mathrm{M} 3 \mathrm{CI} / \mathrm{ABS}(\mathrm{M} 3 \mathrm{CI}) \\
& \quad=\operatorname{SQRT}\left(\left(\delta \mathrm{Num} /(\mathrm{Num})^{2}+(\delta \text { Den } / \text { Den })^{2}\right)\right.
\end{aligned}
$$

where $\delta R_{\mathrm{R}}, \delta R_{\mathrm{RE}}$, and $\delta R_{\mathrm{NIR}}$ represent the uncertainties in the red $(660 \mathrm{~nm})$, red-edge (710 or $720 \mathrm{~nm}$ ), and near infrared (NIR) $(810 \mathrm{~nm})$ bands, respectively. For a given estimated uncertainty in each of these bands, the uncertainty in the numerator ( $\delta \mathrm{Num})$ and denominator ( $\delta$ Den) are used to compute the uncertainty in $\mathrm{M} 3 \mathrm{CI}(\delta \mathrm{M} 3 \mathrm{CI})$.

An uncertainty analysis was performed for the leaf reflectance measurements, following Perry and Davenport (2007). The improvement in $\% \mathrm{~N}$ estimation with increasing sample size was evaluated by varying the number of the number of leaves averaged to represent a sample from 1 to 10 . Each of the sample subsets (one leaf, two leaves, etc.) were used to estimate $\% \mathrm{~N}$, based on relationships presented in the Results, and these estimates were compared with the measured leaf $\% \mathrm{~N}$ for that sample.

\section{Results}

Leaf $\% N$ trends. The leaf $\% \mathrm{~N}$ results are summarized in Fig. 2. The overall range of $\% \mathrm{~N}$ spanned from slightly greater than $1.7 \%$ to almost $4 \%$. There were strong seasonal trends, with leaf $\% \mathrm{~N}$ values highest at the beginning of the season and smallest after harvest. The range of leaf $\% \mathrm{~N}$ over time was much greater than differences across treatments.

Leaf $\% N$ from canopy reflectance. Regression results for leaf $\% \mathrm{~N}$ on each of the canopy spectral indices are shown in Fig. 3. M3CI_710 $\mathrm{nm}$ resulted in the highest $R^{2}$ value $(0.67)$ with measured leaf $\% \mathrm{~N}$, followed by CCCI_710 nm and SCCCI_710 nm $\left(R^{2}=0.66\right)$. For the indices CCCI, SCCCI, and M3CI, the use of the 710-nm band yielded higher $R^{2}$ values than using the 720$\mathrm{nm}$ band. For NDRE and CI, the $720-\mathrm{nm}$ band resulted in higher $R^{2}$ values. The indices MCARI and NDVI showed little or no correlation with measured leaf $\% \mathrm{~N}$. All of the regressions were significant at $P<0.001$ except for MCARI and NDVI. The RMSE values ranged from $0.24 \% \mathrm{~N}$ for $\mathrm{M} 3 \mathrm{CI}$ to $0.30 \% \mathrm{~N}$ for CI_710 $\mathrm{nm}$.

Leaf $\% N$ from leaf reflectance. The regression results of leaf $\% \mathrm{~N}$ on the suite of spectral indices are shown in Table 2. For the indices measured at both canopy and leaf scales, the results are somewhat different from those from the canopy-scale measurements. NDVI and NDRE $\left(R^{2}=0.65\right)$ performed as well or better than CCCI_720 nm or M3CI $710 \mathrm{~nm}\left(R^{2}=0.61\right)$. For the indices computed only on leaf reflectance, the $R^{2}$ values ranged from $R^{2}=0.52$ (MCARI) to 0.00 (PSRI).

Error analyses. The error propagation rules presented in the Materials and Methods were used to determine the effects of uncertainty in canopy reflectance measurements on leaf $\% \mathrm{~N}$. Uncertainty in canopy reflectance was varied from \pm 0.01 to \pm 0.05 and the corresponding uncertainty in M3CI_710 nm was computed. The uncertainty in leaf $\% \mathrm{~N}$ was determined as the product of the uncertainty in M3CI_710 nm and the slope of the regression model (4.87; Fig. 3C) relating M3CI_710 to leaf $\% \mathrm{~N}$. For uncertainty in canopy reflectance ranging from 0.01 to 0.05 , the resulting uncertainty in leaf $\% \mathrm{~N}$ was $0.2-0.8 \% \mathrm{~N}(\mathrm{M} 3 \mathrm{CI}=0.5)$ and from 0.2 to $1.1 \% \mathrm{~N}(\mathrm{M} 3 \mathrm{CI}=0.8)$.

The uncertainty analysis for leaf reflectance sample size on leaf $\mathrm{N}$ was performed as described in the Materials and Methods for the indices NDVI and M3CI. The regression models presented in Table 2 were used to determine leaf $\% \mathrm{~N}$ for each averaged value of the index (each subset). The resulting graph of delta leaf $\% \mathrm{~N}$ by number of leaves is shown in Fig. 4. Note that the difference between the measured and estimated leaf $\% \mathrm{~N}$ decreases as the number of leaves approaches 10. Unfortunately, the size of the dataset did not allow for averaging of more than 10 leaves.

\section{Discussion}

Results at the canopy scale indicate that CCCI, SCCCI, and M3CI using the 710-nm band for red-edge reflectance produced similar results. SCCCI has the advantage over $\mathrm{CCCI}$ in that it does not require bounds to be computed. Hence, each dataset can be treated independently, with no requirements for pooling of data to get a sufficient range of NDVI and NDRE values to determine the relationship. $\mathrm{M} 3 \mathrm{CI}$ has the additional advantage in that it reduces SCCCI from eight terms and three ratios to six terms and one ratio. This has implications for error propagation (e.g., reflectance uncertainty) through the index. Note that the CI $720 \mathrm{~nm}$ and NDRE_720 $\mathrm{nm}$ resulted in $R^{2}$ values only slightly lower than CCCI, SCCCI, and M3CI (e.g., 0.59 vs. 0.67 for CI_720 nm and M3CI, respectively). The $\mathrm{CI}$ index has two terms and

\begin{tabular}{|c|c|c|c|c|c|}
\hline \multicolumn{6}{|c|}{ Red-blush pear, Yr $3 ; N=35$} \\
\hline Index & Intercept (s.e.) & Slope (s.e.) & $\operatorname{Adj} . R^{2}$ & F Pr & $\operatorname{RMSE}(\% \mathrm{~N})$ \\
\hline$\overline{\text { CCCI_720 nm }}$ & $3.17(0.12)$ & $3.45(0.47)$ & 0.61 & $<0.001$ & 0.28 \\
\hline CI_720 nm & $-0.27(0.39)$ & $14.71(2.16)$ & 0.57 & $<0.001$ & 0.29 \\
\hline EVI & $5.33(0.55)$ & $-93.4(17.2)$ & 0.46 & $<0.001$ & 0.33 \\
\hline M3CI_710 nm & $-2.46(0.66)$ & $10.3(1.4)$ & 0.61 & $<0.001$ & 0.28 \\
\hline M3CI_720 nm & $-2.2(0.68)$ & $14.67(2.17)$ & 0.57 & $<0.001$ & 0.29 \\
\hline M3CI_730 nm & $-2.37(1.24)$ & $31.09(8.15)$ & 0.29 & $<0.001$ & 0.37 \\
\hline MCARI & $3.73(0.23)$ & $-8.6(1.40)$ & 0.52 & $<0.001$ & 0.31 \\
\hline ModCAI & $1.78(0.57)$ & $-65.4(64.10)$ & 0.10 & $<0.001$ & 0.44 \\
\hline NDRE_720 nm & $-0.91(0.41)$ & $14.82(1.85)$ & 0.65 & $<0.001$ & 0.26 \\
\hline NDRE_720 nm/NDVI & $-1.17(0.49)$ & $12.77(1.75)$ & 0.61 & $<0.001$ & 0.28 \\
\hline NDVI & $-21.21(2.94)$ & $29.54(3.68)$ & 0.65 & $<0.001$ & 0.26 \\
\hline PSRI & - & - & 0.00 & - & - \\
\hline SIPI & $64.6(12.5)$ & $-61.3(12.3)$ & 0.41 & $<0.001$ & 0.34 \\
\hline TGI & $3.91(0.37)$ & $-0.37(-0.37)$ & 0.34 & $<0.001$ & 0.36 \\
\hline
\end{tabular}
one ratio, which may result in lower errors

Table 2. Regression coefficients and statistics for leaf $\% \mathrm{~N}$ against indices from leaf reflectance using a portable spectrometer.

$\mathrm{CCCI}=$ Canopy Chlorophyll Content Index; CI = Chlorophyll Index; EVI = Enhanced Vegetation Index; M3CI = Modified Canopy Chlorophyll Content Index; MCARI = Modified Chlorophyll Absorption Reflectance Index; NDRE = Normalized Difference Red-edge Index; NDVI = Normalized Difference Vegetation Index; PSRI = Plant Senescence Reflectance Index; RMSE = root mean square error; SIPI = Structure Insensitive Pigment Index; TGI = Triangular Greenness Index. 


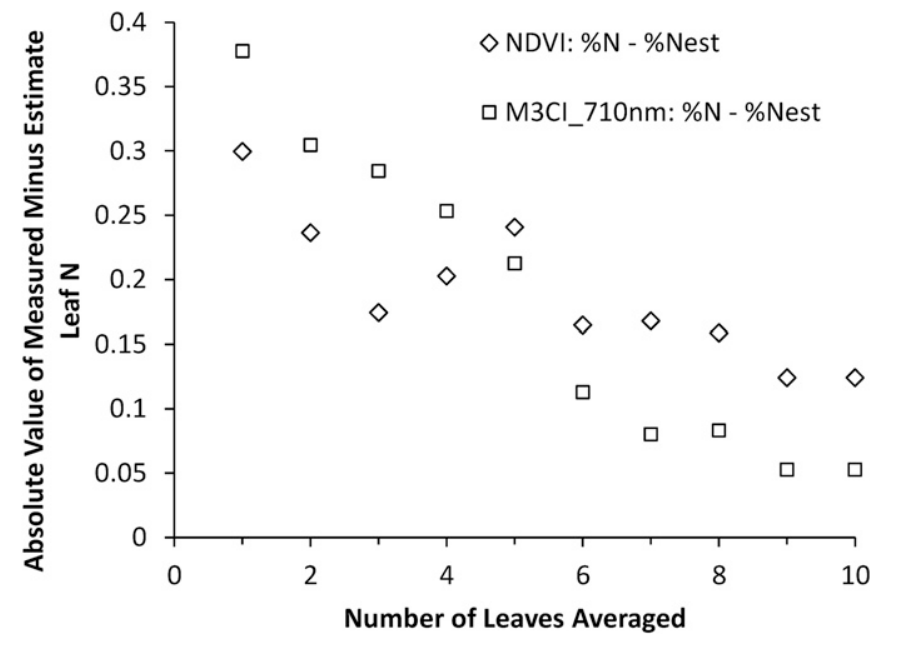

Fig. 4. Leaf $\% \mathrm{~N}$ error (measured minus estimated) as a function of sample size (the number of leaves averaged).

relative to $\mathrm{M} 3 \mathrm{CI}$ when the reflectance uncertainty increases.

Indices with the highest correlation with leaf $\mathrm{N}$ differed between the canopy and leaf reflectance data. There are several factors that can explain these differences. The field of view for the leaf-level and canopy-level measurements were very different. For the canopy-level measurements, while the soil and orchard floor vegetation was masked out, the measurements included leaves at different growth stages, leaves that were damaged, branches, and some shadows. Measurements at the leaf level were biased toward healthy leaves and sampled for $\mathrm{N}$ analysis according to protocols described in the Materials and Methods. CCCI was developed as a canopylevel index (Fitzgerald et al., 2010) to compensate for the effects of biomass saturating the structural index NDVI and to use NDRE to characterize changes in the red-edge (the spectral region bounded by red and NIR wavelengths). NDVI is based on reflectance in the red region, dominated by absorption of light (related to chlorophyll pigments) and in the NIR, dominated by reflectance of the mesophyll tissue. NDRE (as well as chlorophyll indices such as CI) measure subtle changes in the shape of the red-edge reflectance curve. A universal characteristic of leaf spectra is a "blue shift" for plants under stress whereby the red-edge shifts toward shorter wavelengths. The sample diameter for the leaf clip was $\approx 1 \mathrm{~cm}$ and for a single leaf. At this scale, NDVI and simple ratio indices do not saturate. Portable leaf chlorophyll meters (e.g., Minolta SPAD 502; Konica, Osaka, Japan) use measurements of transmitted light in the red and NIR regions to estimate leaf $\% \mathrm{~N}$.

Estimates of leaf $\% \mathrm{~N}$ from canopy and leaf-level reflectance measurements resulted in similar uncertainties (indicated by RMSEs), $<0.3 \% \mathrm{~N}$. These uncertainties are well within the seasonal trends and within the range across treatments for a given date (Fig. 2). Given the high accuracy and control of leaflevel measurements, this indicates that other confounding stresses (abiotic or biotic) are influencing the relationship between the chlorophyll indices and leaf $\% \mathrm{~N}$ for both leaf- and canopy-level reflectance measurements.

To achieve the best estimates of $\% \mathrm{~N}$, the selection of the optimum index (for canopy vs. leaf) and the accuracy of the reflectance measurements are key factors. Canopy reflectance measurements require consistent and accurate calibration to convert the camera or sensor raw data to reflectance values. Results from the error propagation demonstrate that for a value of $\mathrm{M} 3 \mathrm{CI}=0.6$ (close to the mean of the data), the uncertainty in reflectance needs to be less than \pm 0.02 reflectance units to maintain an uncertainty of $0.3 \% \mathrm{~N}$. For the leaf reflectance measurements, the built-in light source and calibration reduce any effects due to changes in irradiance from sunlight. However, one of the challenges of leaf-level measurements is that the estimation accuracy generally improves with sample size. For example, Perry and Davenport (2007) demonstrated an increase in estimation accuracy of leaf $\% \mathrm{~N}$ in apple as the number of leaves averaged approached 20 . The uncertainty analysis performed using NDVI and M3CI_710 nm (Fig. 4) indicated that 10 leaves or more per tree would be a target number to average for consistent results.

The results support the use of either canopy or leaf-level reflectance measurements to estimate leaf $\% \mathrm{~N}$ in red-blush pear. Given the number of measurements required (10 or more per tree), leaf-level measurements are not practical for characterizing variability across an orchard; however, canopy-level measurements made from UAVs offer the potential for spatial mapping of leaf $\% \mathrm{~N}$. The first industry application of $\mathrm{N}$ estimates from UAVs is likely to be an orchard block estimate of $\mathrm{N}$ with summary statistics such as average, minimum, and maximum. However, the canopy $\mathrm{N}$ maps generated from the UAV imagery could be highly valuable as related technologies develop. Some examples include foliar applications allowing variable rates of $\mathrm{N}$ and use of tree-scale precision fertigation.

\section{Literature Cited}

Allen, R., L.S. Pereira, D. Raes, and M. Smith. 1998. Crop evapotranspiration-Guidelines for computing crop water requirements. FAO irrigation and drainage paper 56. FAO, Rome, Italy.

Australian Bureau of Statistics (ABS). 2016a. Agricultural commodities, Australia, 201415, Cat. no. 7121.0. Australian Bureau of Statistics, Canberra, Australia. 27 Dec. 2017. $<$ http://www.abs.gov.au>.

Australian Bureau of Statistics (ABS). 2016b. Value of agricultural commodities produced, Australia, 2014-15, Cat. no. 7503.0. Australian Bureau of Statistics, Canberra, Australia. 27 Dec. 2017. <http://www.abs.gov.au>.

Ball, G.H. and D.J. Hall 1965. Isodata: A method of data analysis and pattern classification. Stanford Research Institute, Menlo Park, CA.

Barnes, E.M., T.R. Clarke, and S.E. Richards. 2000. Coincident detection of crop water stress, nitrogen status and canopy density using ground based multispectral data. Fifth International Conference on Precision Agriculture, Madison, WI

Brunetto, G., G.W. Bastos de Melo, M. Toselli, M. Quartieri, and M. Tagliavini. 2015. The role of mineral nutrition on yields and fruit quality in grapevine, pear and apple. Rev. Bras. Frutic. 37:1089-1104.

Bureau of Meteorology. 2017. Climate Data Online, Australian Government, Bureau of Meteorology, Product Code: IDCJAC0001 reference: 31092264.

Clarke, T.R., M.S. Moran, E.M. Barnes, P.J. Pinter, and J. Qi. 2001. Planar domain indices: A method for measuring a quality of a single component in two-component pixels. IEEE International Geoscience and Remote Sensing Symposium, Sydney, Australia, 9-13 July 2001.

Curetti, M., E. Sánchez, M. Tagliavini, and P. Gioacchini. 2013. Foliar-applied urea at bloom improves early fruit growth and nitrogen status of spur leaves in pear trees, cv. Williams bon chretien. Sci. Hort. 150:16-21.

Daughtry, C.S.T. 2001. Discriminating crop residues from soil by shortwave infrared reflectance. Agron. J. 93:125-131.

Daughtry, C.S.T., C.L. Walthall, M.S. Kim, E.B. De Colstoun, and J.E. Mcmurtrey, III. 2000. Estimating corn leaf chlorophyll concentration from leaf and canopy reflectance. Remote Sens. Environ. 74:229-239.

Fitzgerald, G., D. Rodriguez, and G. O'leary. 2010. Measuring and predicting canopy nitrogen nutrition in wheat using a spectral indexThe canopy chlorophyll content index (CCCI). Field Crops Res. 116:318-324.

Gitelson, A.A., G.P. Keydan, and M.N. Merzlyak. 2006. Three-band model for noninvasive estimation of chlorophyll, carotenoids, and anthocyanin contents in higher plant leaves. Geophys. Res. Lett. 33:1-5.

Goodwin, I., S. Green, and D. Cornwall. 2015. Transpiration of pear trees and implications for irrigation scheduling. Acta Hort. 1094:317324.

Huete, A., K. Didan, T. Miura, E.P. Rodriguez, X. Gao, and L.G. Ferreira. 2002. Overview of the radiometric and biophysical performance of the MODIS vegetation indices. Remote Sens. Environ. 83:195-213. 
Human, J.P. 2013. Breeding blush pears (Pyrus Communis L.) in South Africa. Acta Hort. 976:383-388.

Hunt, Jr., E.R., P.C. Doraiswamy, J.E. Mcmurtrey, C.S.T. Daughtry, E.M. Perry, and B. Akhmedov. 2013. A visible band index for remote sensing leaf chlorophyll content at the canopy scale. Intl. J. Appl. Earth Obs. Geoinf. 21:103112.

Isbell, R.F. 2002. The Australian soil classification. CSIRO Publishing, Clayton, Australia.

Jie, W., Z. Hua-Bing, S. Chang-Wei, C. Qiao-Wei, D. Cai-Xia, and X. Yang-Chun. 2014. Determination of nitrogen concentration in fresh pear leaves by visible/near-infrared reflectance spectroscopy. Agron. J. 106:1867-1872.

Merzlyak, M.N., A.A. Gitelson, O.B. Chivkunova, and V.Y.U. Rakitin. 1999. Non-destructive optical detection of pigment changes during leaf senescence and fruit ripening. Physiol. Plant. 106:135-141.
Penuelas, J., F. Baret, and I. Filella. 1995. Semiempirical indices to assess carotenoids/chlorophyl a ratio from leaf spectral reflectance. Photosynthetica 31:221-230.

Perry, E.M., G.J. Fitzgerald, J.G. Nuttall, G.J. O'leary, U. Schulthess, and A. Whitlock. 2012. Rapid estimation of canopy nitrogen of cereal crops at paddock scale using a canopy chlorophyll content index. Field Crops Res. 134:158-164.

Perry, E.M. and J.R. Davenport. 2007. Spectral and spatial differences in response of vegetation indices to nitrogen treatments on apple. Comput. Electron. Agr. 59:56-65.

R Core Team. 2017. R: A language and environment for statistical computing. R Foundation for Statistical Computing, Vienna, Austria. 27 Dec. 2017. <https://www.R-project.org/>.

Reuter, D.J. and J.B. Robinson. 1997. Plant analysis: An interpretation manual. CSIRO Publishing, Collingwood, Australia.
Rouse, Jr., J.W., R.H. Haas, J.A. Schell, and D.W. Deering. 1973. Monitoring vegetation systems in the Great Plains with ETRS. Earth Res. Tech. Satellite-1 Symp., Goddard Space Flight Cent., Washington, DC, 10-14 Dec. 1973. p. 309 317.

Sánchez, E.E., M. Curetti, and D. Sugar. 2008. Foliar application of urea during bloom increases fruit size in 'Williams' pears. Acta Hort. 800:583-586.

Sugar, D., T.L. Righetti, E.E. Sanchez, and H. Khemira. 1992. Management of nitrogen and calcium in pear trees for enhancement of fruit resistance to postharvest decay. HortTechnology 2:382-387.

Wang, H. and L. Cheng. 2011. Differential effects of nitrogen supply on skin pigmentation and flesh starch breakdown of 'Gala' apple. HortScience 46:1116-1120. 